\title{
Identification of transcription factors and single nucleotide polymorphisms of Lrh1 and its homologous genes in Lrh1-knockout pancreas of mice
}

Maochun Tang ${ }^{1 \dagger}$, Li Cheng ${ }^{2 \dagger}$, Rongrong Jia', Lei Qiu', Hua Liu', Shu Zhou', Xiuying Ma', Guoyong Hu², Xingpeng Wang ${ }^{2}$ and Yan Zhao ${ }^{1 *}$

\begin{abstract}
Background: To identify transcription factors (TFs) and single nucleotide polymorphisms (SNPS) of Lrh1 (also named Nr5a2) and its homologous genes in Lrh1-knockout pancreas of mice.

Methods: The RNA-Seq data GSE34030 were downloaded from Gene Expression Omnibus (GEO) database, including 2 Lrh1 pancreas knockout samples and 2 wild type samples. All reads were processed through TopHat and Cufflinks package to calculate gene-expression level. Then, the differentially expressed genes (DEGs) were identified via non-parametric algorithm (NOISeq) methods in R package, of which the homology genes of Lrh1 were identified via BLASTN analysis. Furthermore, the TFs of Lrh1 and its homologous genes were selected based on TRANSFAC database. Additionally, the SNPs were analyzed via SAM tool to record the locations of mutant sites.

Results: Total 15683 DEGs were identified, of which 23 was Lrh 1 homology genes (3 up-regulated and 20 down-regulated). Fetoprotein TF (FTF) was the only TF of Lrh1 identified and the promoter-binding factor of FTF was CYP7A. The SNP annotations of Lrhl homologous genes showed that 92\% of the mutation sites were occurred in intron and upstream. Three SNPS of Lrh1 were located in intron, while 1819 SNPs of Phkb were located in intron and 1343 SNPs were located in the upstream region.

Conclusion: FTF combined with CYP7A might play an important role in Lrh1 regulated pancreas-specific transcriptional network. Furthermore, the SNPs analysis of Lrh1 and its homology genes provided the candidate mutant sites that might affect the Lrh1-related production and secretion of pancreatic fluid.
\end{abstract}

Keyword: Lrh1-knockout pancreas, RNA-Seq, Lrh1 homologous gene, Transcription factor, Single nucleotide polymorphisms

\section{Background}

The pancreas is an endocrine gland, producing insulin, glucagon, somatostatin, and pancreatic polypeptide, and also an exocrine gland, accounting for more than $98 \%$ of pancreatic gland and secreting pancreatic juice containing digestive enzymes [1]. These digestive enzymes help to further break down the carbohydrates, proteins and

\footnotetext{
* Correspondence: 320zhaoyan@163.com

${ }^{\dagger}$ Equal contributors

'Department of Gastroenterology, Shanghai Tenth People's Hospital, Tongji University School of Medicine, No.301, Yanchang Middle Road, Shanghai 200072, China

Full list of author information is available at the end of the article
}

lipids in the chime and thus support the absorption and digestion of nutrition in small intestine [2]. In the past decades, many research have focused on target genes and transcription factors (TFs) involved in the exocrine pancreas-specific transcriptional networks which are required for the production and secretion of pancreatic fluid that helps out the digestive system. Currently, many exocrine pancreas-specific genes and transcription factors have been identified, which may promote the understanding of the effect of exocrine pancreas on digestive system.

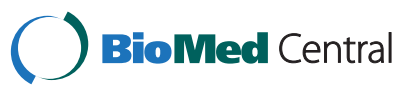

(c) 2014 Tang et al.; licensee BioMed Central Ltd. This is an Open Access article distributed under the terms of the Creative Commons Attribution License (http://creativecommons.org/licenses/by/2.0), which permits unrestricted use, distribution, and reproduction in any medium, provided the original work is properly credited. The Creative Commons Public Domain Dedication waiver (http://creativecommons.org/publicdomain/zero/1.0/) applies to the data made available in this article, unless otherwise stated. 
Liver receptor homolog-1 (Lrh1; also called Nr5a2) is a nuclear receptor of ligand-activated transcription factors in liver by binding as a monomer to DNA sequence elements with the consensus sequence $5^{\prime}-\mathrm{Py}$ CAAGGPyCPu-3' [3]. It has been suggested that Lrh1 is progressively expressed in both the endocrine and exocrine pancreas [4]. Baquié $M$ et al. [5] have found that Lrh1 is expressed in human islets and protects $\beta$-cells against stress-induced apoptosis that may be mediated via the increased glucocorticoid production that blunts the pro-inflammatory response of islets. Meanwhile, Fayard E et al. [6] have demonstrated that both Lrh1 and CEL (encoding carboxyl ester lipase) are co-expressed and confined to the exocrine pancreas. The identification of $C E L$ as an $L r h 1$-target gene indicates that $L r h 1$ plays an important role in enterohepatic cholesterol homeostasis associated with the absorption of cholesteryl esters and the assembly of lipoproteins by the intestine [7]. Besides, Lrh1 is a downstream target in the PDX-1 (lead to pancreas agenesis) regulatory cascade that is activated only during early stages of pancreas development and that governs pancreatic development, differentiation and function [8].

Recently, the rapid advent of next-generation sequencing has made this technology broadly available for researchers in various molecular and cellular biological fields. Holmstrom SR et al. [9] have determined the cistrome and transcriptome for the nuclear receptor LRH-1 in exocrine pancreas and revealed that Lrh1 directly induces expression of genes encoding digestive enzymes and secretory and mitochondrial proteins based on Chromatin immunoprecipitation (ChIP)-seq and RNAseq analyses. Besides, Lrh1 cooperates with the pancreas transcription factor 1-L complex (PTF1-L) in regulation of exocrine pancreas-specific gene expression. However, many potential target genes and TFs of Lrh1 based on RNA-seq analysis have not been revealed.

In the present study, we downloaded the raw RNA-seq data of Holmstrom SR et al. deposited in The National Center for Biotechnology Information (NCBI) database, which were analyzed using multiple bioinformatics tools in the purpose of finding specific TFs of Lrh1 and its homology genes. Additionally, we also annotated the SNPs of Lrh1 and its homology genes to predict their mutant sites. Our study might improve the understanding of the regulation network of Lrh1-related production and secretion of pancreatic fluid.

\section{Methods}

\section{RNA-seq data acquisition}

The RNA-seq data was downloaded from NCBI (http://www.ncbi.nlm.nih.gov/) Gene Expression Omnibus (GEO) database (GEO accession: GSE34030 [9]), including 2 Lrh1 pancreas knockout samples and 2 wild type samples. RNA preparations were subjected to the Illumina RNA-seq protocol and the platform was GPL9185.

\section{Data pre-processing, gene expression and homology gene of $\operatorname{Lrh} 1$}

The raw data were downloaded from SRA (Sequence Read Archive) of NCBI and then converted to fastq reads using fastq-dump program of NCBI SRA Toolkit (-q 64) (http://trace.ncbi.nlm.nih.gov/Traces/sra/sra.cgi?view=std). Then, these reads were processed through TopHat [10] and Cufflinks [11] package to calculate gene-expression level. All parameters were set up according to the default settings of TopHat and Cufflinks. The DEGs were identified via non-parametric algorithm (NOISeq) methods in $\mathrm{R}$ package [12]. The thresholds value was False Discovery Rate $($ FDR $)<0.001$. BLASTN analysis $[13,14]$ of the selected DEGs was used to identify the homology genes of Lrh1. Homology genes here refer to the paralogous genes which share a high degree of sequence similarity (maximum expectation value was set to $\mathrm{e}^{-5}$ ) with $\operatorname{Lrh} 1$ in mice.

\section{Function annotation of $L$ rh1 homologous genes}

For functional analysis of Lrh1 homologous genes, DAVID (Database for Annotation, Visualization and Integrated Discovery) [15] was performed for Gene Ontology (GO) [16] function and Kyoto Encyclopedia of Genes and Genomes (KEGG) pathway enrichment analysis.

\section{Transcription factor (TF) of $L r h 1$ homologous genes}

Combined with TRANSFAC database [17], the TFs regulated the transcription of Lrh1 and its homologous genes were identified. Then, the promoter-binding factors regulated via the selected TFs were analyzed based on the website (http://www.nursa.org/molecule.cfm?molType= receptor\&molId $=5 \mathrm{~A} 2$ ).

\section{Screening of SNPs}

The fastq reads were mapped to marker sequences using bowtie [18]. And the aligned reads were called using the SAM tool [19]. In order to minimize the risk of false-positive SNP Callings, the threshold value was that ID was “":" with quality $>50$, or ID was not "*" with quality $>20$. These SNPs were annotated via SnpEff [20] to categorize the effects of variants in genome sequences. The identified SNPs were searched in the dbSNP database to identify diseased SNPs or de novo discovered SNPs.

\section{Results}

Identification and homology analysis of differentially expressed genes

After data processing, at FDR $<0.001$, a total of 15683 DEGs were identified, including 10994 up-regulated and 4698 down-regulated genes. BLASTN analysis of DEGs showed 23 Lrh1 homology genes. Among them, 3 were up-regulated and 20 were down-regulated (Table 1). 
Table 1 Lrh1 homology genes of differentially expressed genes

\begin{tabular}{ll}
\hline Regulation & Lrh1 homology genes \\
\hline Up-regulated & Fzd5, Klrb1f, Phc1 \\
Down-regulated & March8, Zfp282, Atrx, Sos2, Zfc3h1, Pofut1, Gm9079, \\
& $\begin{array}{l}\text { Ykt6, Phkb, Galc, Setd1a, Fzd5, Kazn, Kcnmb1, Lamc2, } \\
\text { Mylk4, Ache, Pbxip1, Lrh1, Phc1 }\end{array}$ \\
\hline
\end{tabular}

Function and pathway annotation of $L r h 1$ homologous genes To determine the function of Lrh1 homologous genes in pancreas, GO enrichment analysis and KEGG pathway enrichment analysis were used to analyze the up- and down-regulated Lrh1 homologous genes. For function and pathway annotation, DEGs were enriched into hexose metabolic process and monosaccharide metabolic process, which were involved into glycometabolism (Figure 1). Meanwhile, KEGG pathway enrichment analysis identified insulin signaling pathway, indicating that the disorders of glycometabolism might be resulted from insulin resistance and/or insulin secretion (Figure 2). PHKB, an Lrh1 homologous gene, participated in GO terms (hexose metabolic process and monosaccharide metabolic process) and KEGG pathway (insulin signaling pathway), was identified.

\section{Potential TFs of Lrh1 homologous genes}

Fetoprotein transcription factor (FTF) (ID: T04754) of Lrh1 was the only TF identified based on TRANSFAC database.
Meanwhile, the promoter-binding factor of $\operatorname{Lrh} 1$ was CYP7A (Cholesterol 7 $\alpha$-hydroxylase).

\section{SNPs of Lrh1 homologous genes}

The annotation of SNPs of Lrh1 homologous genes showed that the majority of SNPs were located in intron and upstream, accounting for nearly 92\% of all SNPs (Tables 2 and 3). Three SNPs of Lrh1 were distributed in intron. Meanwhile, total 1819 SNPs of $P h k b$ were located in the intron and 1343 SNPs were located in the upstream region of $P h k b$.

\section{Discussion}

In the present study, combined with RNA-seq data of Lrh1-knockout pancreas samples, FTF was the only TF of Lrh1 identified based on TRANSFAC database and may regulate cholesterol catabolism into bile acids by activation of the promoter-binding factor CYP7A. Many literatures have elucidated the function of Lrh1/Nr5a2/FTF/CYP7A via experimental studies [21-25].

FTF is highly expressed in the liver and intestine and is implicated in the regulation of cholesterol, bile acid and steroid hormone homeostasis [26]. Nearly $50 \%$ of the body cholesterol is catabolized to bile acids via bile acid biosynthetic pathway, of which cholic acid (hydroxylated at position 12) and chenodeoxycholic acid are the major primary bile acids and play an important

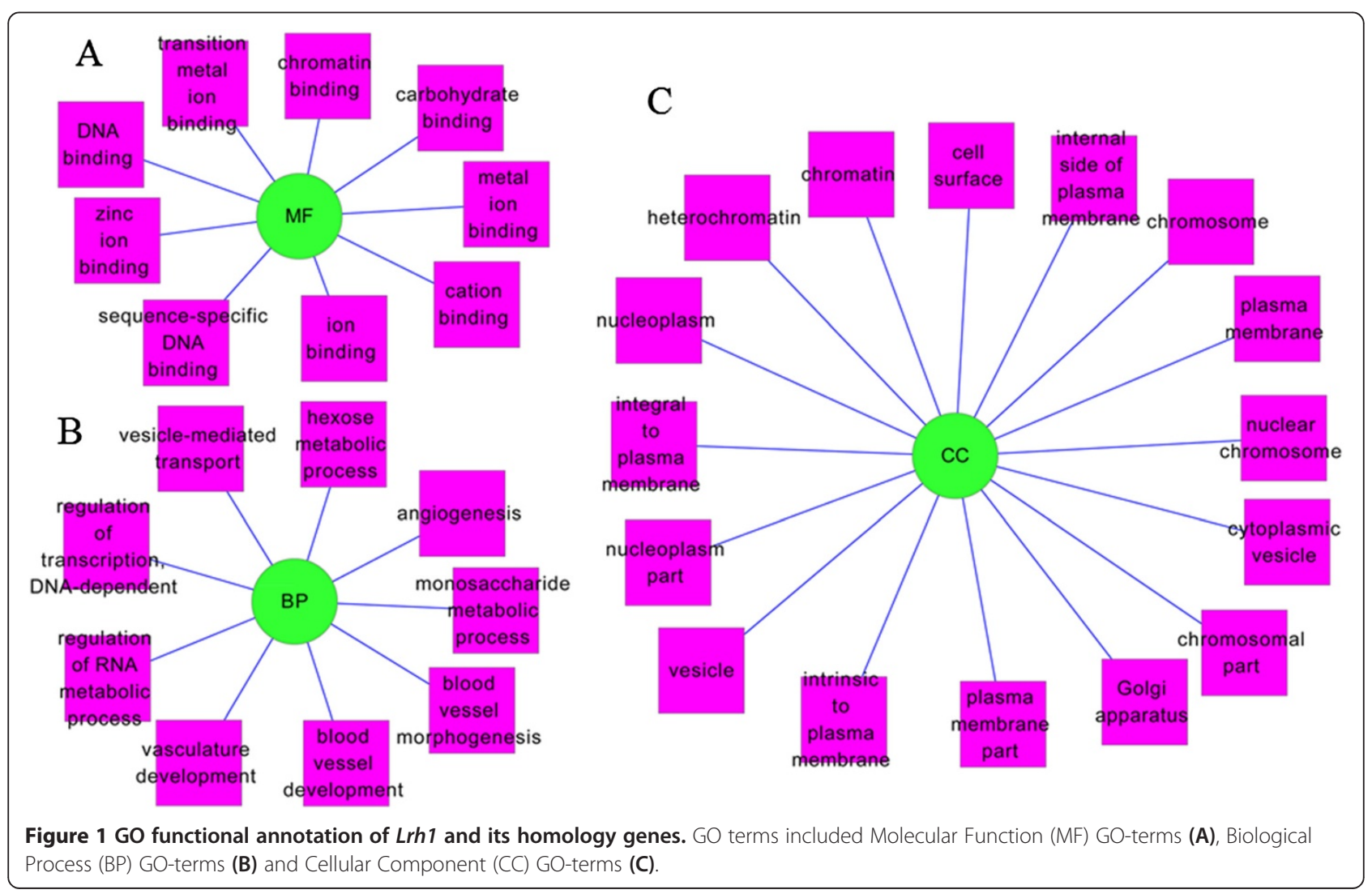




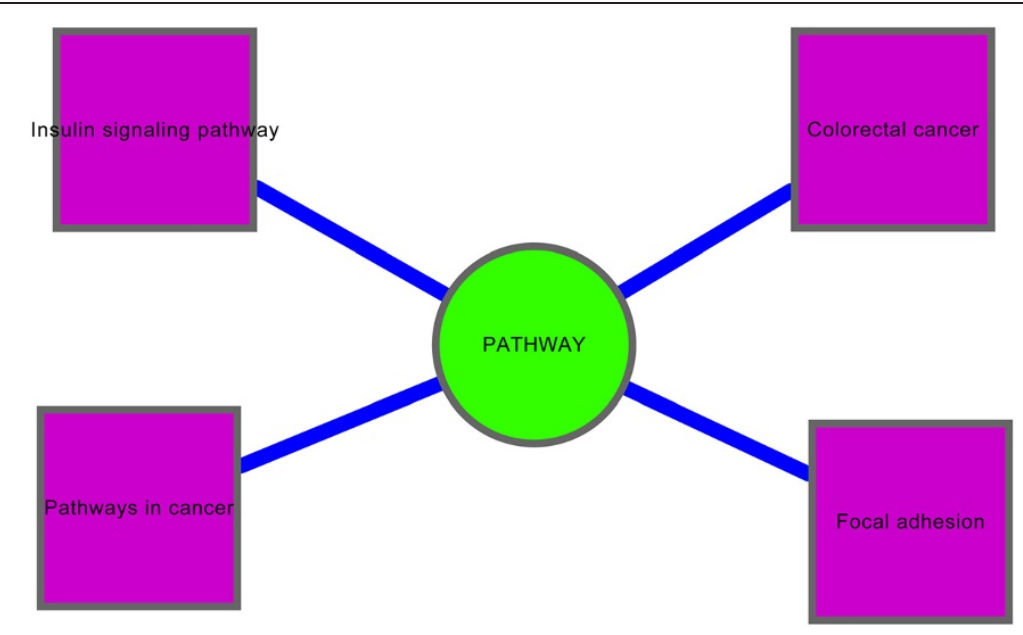

Figure 2 KEGG pathway annotations of $L r h 1$ and its homology genes.

role in cholesterol homeostasis [19]. Chenodeoxycholic acid can repress FTF expression and is a more potent suppressor of HMG-CoA reductase and cholesterol $7 \alpha$-hydroxylase/CYP7A1 (7 $\alpha$-hydroxylase) than cholic acid [27]. It has been proposed that Lrh1, also known as CYP7A promoter-binding factor, $L R H 1$, or FTF, is required for the transcription of the $7 \alpha$-hydroxylase gene $[19,28]$. The small heterodimer partner 1 (SHP) of the nuclear bile acid receptor, FXR (farnesoid X receptor) can dimerize with FTF and diminish its activity on the $7 \alpha$-hydroxylase promoter [29].

Although Lrh1 has been demonstrated the function in feedback regulation of CYP7A1 expression as part of the FXR-SHP-LRH-1 cascade, in which bile acids can inhibit their own synthesis, the mechanisms have not been well understood. Out $C$ et al. [25] have suggested that CYP7A1 expression is increased rather than decreased under chow-fed conditions in Lrh1-knockdown mice that is coincided with a significant reduction in expression of intestinal Fgf15, a suppressor of CYP7A1. Besides, Noshiro $\mathrm{M}$ et al. [30] have suggested that the circadian rhythm of

Table 2 The number of different type of SNPs of $L r h 1$ and its homology genes

\begin{tabular}{lll}
\hline Type & Number & Proportion \\
\hline Downstream & 581 & 0.052234 \\
Intron & 8251 & 0.741796 \\
Non-synonymous-coding & 122 & 0.010968 \\
Splice-site-donor & 2 & 0.00018 \\
Start-gained & 4 & 0.00036 \\
Stop-gained & 5 & 0.00045 \\
Synonymous-coding & 97 & 0.008721 \\
Upstream & 1975 & 0.17756 \\
UTR-3-prime & 79 & 0.007102 \\
UTR-5-prime & 7 & 0.000629 \\
\hline
\end{tabular}

CYP7A is regulated by multiple transcription factors, including DBP, REV-ERB $\alpha / \beta$, LXR $\alpha, \mathrm{HNF} 4 \alpha$ DEC2, E4BP4, and PPAR $\alpha$. Hepatocyte nuclear factor $4 \alpha$ (HNF4 $\alpha$ ) and FTF are two major TFs driving CYP7A1 promoter activity in lipid homeostasis. Bochkis IM et al. [31] have shown that prospero-related homeobox (Prox1) directly interacts with both HNF4 $\alpha$ and FTF and potently co-represses CYP7A1 transcription.

In the present study, we annotated the SNPs of Lrh1 and its homologous genes, showing that the majority was located in intron and upstream. Quiles Romagosa MÁ [32] has reported that a functional SNP located in Lrh1 promoter is related to Body Mass Index (BMI) and these SNPs might play important roles in the obese phenotype. However, previous researches mostly focused on SNPs associated with pancreatic cancer cell growth

Table 3 The annotation of SNPs of Lrh1 and its homology genes

\begin{tabular}{llll}
\hline Gene name & Chromo & Number & Position \\
\hline Zfc3h1 & 10 & 4 & 115385510 \\
Galc & 12 & 36 & $98232394-98232429$ \\
Mylk4 & 13 & 13 & $32711300-32711312$ \\
Fzd5 & 1 & 22 & $64737712-64737722$ \\
Lrh1 & 1 & 6 & $136849713-136870578$ \\
Lamc2 & 1 & 923 & $153145967-153191429$ \\
Pofut1 & 2 & 188 & $153239719-153275253$ \\
Ache & 5 & 19 & $137288432-137288449$ \\
Zfp282 & 6 & 1016 & $47876551-47913485$ \\
8-Mar & 6 & 4231 & $116342541-116360897$ \\
Setd1a & 7 & 1834 & $127778726-127802950$ \\
Phkb & 8 & 3197 & $85837045-86008353$ \\
Atrx & $X$ & 7 & $105863283-105906922$ \\
\hline
\end{tabular}


and proliferation. For example, a previous genome-wide association study has identified five SNPs on 1q32.1 associated with pancreatic cancer that mapped to Lrh1 gene and its up-stream regulatory region [33].

\section{Conclusions}

In conclusion, FTF combined with $C Y P 7 A$ might play an important role in $L r h 1$ regulated pancreas-specific transcriptional network. Furthermore, the SNPs analysis of Lrh1 and its homology genes provided the candidate mutant sites that might affect the Lrh1-related production and secretion of pancreatic fluid. These common susceptibility loci for Lrh1 and its homologous genes needed follow-up studies.

\section{Highlights}

1. Total 15683 DEGs were identified, of which 23 was Lrh1 homology genes (3 up-regulated and 20 down-regulated).

2. Fetoprotein TF was the only TF of $L r h 1$ identified based on TRANSFAC database and the promoterbinding factor of fetoprotein TF was CYP7A.

3. The SNP annotations of Lrh1 homologous genes showed that $92 \%$ of mutation sites were occurred in intron and upstream. Three SNPs of $L r h 1$ were located in intron, while 1819 SNPs of $P h k b$ were located in intron and 1343 SNPs were located in upstream region.

\section{Competing interest}

The authors declare that they have no competing interests.

\section{Authors' contributions}

MT, XM and CL participated in the design of this study, and they both performed the statistical analysis. RJ, GYH and YZ carried out the study, together with LQ, collected important background information, and drafted the manuscript. HL, XPW and ZS conceived of this study, and participated in the design and helped to draft the manuscript. All authors read and approved the final manuscript.

\section{Acknowledgements}

This study was supported by grants from the National Natural Science Foundation of China (No. 81200320, 81300350), Shanghai Science and Technology Commission (No. 11JC1410000), Fund of Shanghai Health Bureau (No. 20114315) and Training Plan of Excellent Academic Researcher of Shanghai Tenth People's Hospital (No. 12XSGG105,No. 04.01.13037).

\section{Author details}

${ }^{1}$ Department of Gastroenterology, Shanghai Tenth People's Hospital, Tongji University School of Medicine, No.301, Yanchang Middle Road, Shanghai 200072, China. 'Department of Gastroenterology, Shanghai First People's Hospital Affiliated Shanghai Jiaotong University, Shanghai 200080, China.

Received: 20 December 2013 Accepted: 27 March 2014

Published: 15 April 2014

\section{References}

1. Leung PS: Physiology of the pancreas. In The Renin-Angiotensin System: Current Research Progress in The Pancreas: The RAS in the Pancreas, Volume 690. Netherlands: Springer; 2010:13-27.
2. Whitcomb DC, Lowe ME: Human pancreatic digestive enzymes. Dig Dis Sci 2007, 52:1-17.

3. Fernandez-Marcos PJ, Auwerx J, Schoonjans K: Emerging actions of the nuclear receptor LRH-1 in the gut. Biochimica et Biophysica Acta (BBA)-Molecular Basis of Disease 2011, 1812:947-955.

4. Rausa FM, Galarneau L, Bélanger L, Costa RH: The nuclear receptor fetoprotein transcription factor is coexpressed with its target gene HNF-3 $<\mathrm{i}>\beta</ \mathrm{i}>$ in the developing murine liver intestine and pancreas. Mech Dev 1999, 89:185-188.

5. Baquié M, St-Onge L, Kerr-Conte J, Cobo-Vuilleumier N, Lorenzo PI, Moreno CMJ, Cederroth CR, Nef S, Borot S, Bosco D: The liver receptor homolog-1 $(\mathrm{LRH}-1)$ is expressed in human islets and protects $\beta$-cells against stress-induced apoptosis. Hum Mol Genet 2011, 20:2823-2833.

6. Fayard E, Schoonjans K, Annicotte J-S, Auwerx J: Liver receptor homolog 1 controls the expression of carboxyl ester lipase. J Biol Chem 2003, 278:35725-35731.

7. Hui DY, Howles PN: Carboxyl ester lipase structure-function relationship and physiological role in lipoprotein metabolism and atherosclerosis. J Lipid Res 2002, 43:2017-2030.

8. Annicotte J-S, Fayard E, Swift GH, Selander L, Edlund H, Tanaka T, Kodama T, Schoonjans K, Auwerx J: Pancreatic-duodenal homeobox 1 regulates expression of liver receptor homolog 1 during pancreas development. Mol Cell Biol 2003, 23:6713-6724.

9. Holmstrom SR, Deering T, Swift GH, Poelwijk FJ, Mangelsdorf DJ, Kliewer SA, MacDonald RJ: LRH-1 and PTF1-L coregulate an exocrine pancreas-specific transcriptional network for digestive function. Genes Dev 2011, 25:1674-1679.

10. Trapnell C, Pachter L, Salzberg SL: TopHat: discovering splice junctions with RNA-Seq. Bioinformatics 2009, 25:1105-1111.

11. Trapnell C, Roberts A, Goff L, Pertea G, Kim D, Kelley DR, Pimentel H, Salzberg SL, Rinn JL, Pachter L: Differential gene and transcript expression analysis of RNA-seq experiments with TopHat and Cufflinks. Nat Protoc 2012, 7:562-578.

12. Robles JA, Qureshi SE, Stephen SJ, Wilson SR, Burden CJ, Taylor JM: Efficient experimental design and analysis strategies for the detection of differential expression using RNA-Sequencing. BMC Genomics 2012, 13:484.

13. Altschul SF, Madden TL, Schäffer AA, Zhang J, Zhang Z, Miller W, Lipman DJ: Gapped BLAST and PSI-BLAST: a new generation of protein database search programs. Nucleic Acids Res 1997, 25:3389-3402.

14. Crawford JE, Guelbeogo WM, Sanou A, Traoré A, Vernick KD, Sagnon NF, Lazzaro BP: De novo transcriptome sequencing in Anopheles funestus using Illumina RNA-seq technology. PLoS One 2010, 5:e14202.

15. Da Wei Huang BTS, Lempicki RA: Systematic and integrative analysis of large gene lists using DAVID bioinformatics resources. Nat Protoc 2008, 4:44-57.

16. Hulsegge I, Kommadath A, Smits MA: Globaltest and GOEAST: Two different approaches for Gene Ontology Analysis. BMC Proceedings 2009, 4:S10.

17. Matys V, Kel-Margoulis OV, Fricke E, Liebich I, Land S, Barre-Dirrie A, Reuter I, Chekmenev D, Krull M, Hornischer K: TRANSFAC ${ }^{\circledR}$ and its module TRANSCompel $^{\otimes}$ : transcriptional gene regulation in eukaryotes. Nucleic Acids Res 2006, 34:D108-D110.

18. Langmead B, Trapnell C, Pop M, Salzberg SL: Ultrafast and memory-efficient alignment of short DNA sequences to the human genome. Genome Biol 2009, 10:R25.

19. Gerbod-Giannone M-C, del Castillo-Olivares A, Janciauskiene S, Gil G, Hylemon PB: Suppression of cholesterol 7a-hydroxylase transcription and bile acid synthesis by an a1-antitrypsin peptide via interaction with a1-fetoprotein transcription factor. J Biol Chem 2002, 277:42973-42980.

20. Cingolani P, Platts A, Coon M, Nguyen T, Wang L, Land SJ, Lu X, Ruden DM: A program for annotating and predicting the effects of single nucleotide polymorphisms, SnpEff: SNPs in the genome of Drosophila melanogaster strain w1118; iso-2; iso-3. Fly 2012, 6:80-92.

21. Mouzat K, Baron S, Marceau G, Caira F, Sapin V, Volle DH, Lumbroso S, Lobaccaro JM: Emerging roles for LXRs and LRH-1 in female reproduction. Molecular and cellular endocrinology 2013, 368:47-58.

22. Falender $A E$, Lanz R, Malenfant $D$, Belanger $L$, Richards JS: Differential expression of steroidogenic factor-1 and FTF/LRH-1 in the rodent ovary. Endocrinology 2003, 144:3598-3610.

23. Xu Z, Ouyang L, Castillo-Olivares AD, Pandak WM, Gil G: Alpha(1)-Fetoprotein Transcription Factor (FTF)/Liver Receptor Homolog-1 (LRH-1) Is an Essential Lipogenic Regulator. Biochimica et biophysica acta 1801, 2010:473-479. 
24. del Castillo-Olivares A, Gil G: Alpha 1-fetoprotein transcription factor is required for the expression of sterol 12alpha -hydroxylase, the specific enzyme for cholic acid synthesis. Potential role in the bile acid-mediated regulation of gene transcription. J Biol Chem 2000, 275:17793-17799.

25. Out C, Hageman J, Bloks WW, Gerrits H, Sollewijn Gelpke MD, Bos T, Havinga R, Smit MJ, Kuipers F, Groen AK: Liver receptor homolog-1 is critical for adequate up-regulation of Cyp7a1 gene transcription and bile salt synthesis during bile salt sequestration. Hepatology 2011, 53:2075-2085.

26. Xu Z, Ouyang L, del Castillo-Olivares A, Pandak WM, Gil G: $\mathbf{a}<$ sub $>$ $1</$ sub $>$-Fetoprotein transcription factor (FTF)/liver receptor homolog- 1 (LRH-1) is an essential lipogenic regulator. Biochimica et Biophysica Acto (BBA)-Molecular and Cell Biology of Lipids 2010, 1801:473-479.

27. Fakheri RJ, Javitt NB: Autoregulation of cholesterol synthesis: Physiologic and pathophysiologic consequences. Steroids 2011, 76:211-215.

28. del Castillo-Olivares A, Gil G: Role of FXR and FTF in bile acid-mediated suppression of cholesterol 7a-hydroxylase transcription. Nucleic Acids Res 2000, 28:3587-3593.

29. Del Castillo-Olivares A, Campos JA, Pandak WM, Gil G: Role of FTF/LRH-1 on bile acid biosynthesis. A known nuclear receptor activator that can act as a suppressor of bile acid biosynthesis. J Biol Chem 2004 279(16):16813-16821.

30. Noshiro M, Usui E, Kawamoto T, Kubo H, Fujimoto K, Furukawa M, Honma S, Makishima M, Honma K-i, Kato Y: Multiple mechanisms regulate circadian expression of the gene for cholesterol 7a-hydroxylase (Cyp7a), a key enzyme in hepatic bile acid biosynthesis. J Biol Rhythms 2007, 22:299-311.

31. Bochkis IM, Schug J, Diana ZY, Kurinna S, Stratton SA, Barton MC, Kaestner KH: Genome-wide location analysis reveals distinct transcriptional circuitry by paralogous regulators Foxa1 and Foxa2. PLoS genetics 2012, 8:e1002770

32. Quiles Romagosa MÁ: NR5A2: a regulator of glucose metabolism. 2011, http://repositorio.unican.es/xmlui/bitstream/handle/10902/555/\%5B2\%5D\% 20Quiles\%20Romagosa\%20MA.pdf? sequence $=1$.

33. Petersen GM, Amundadottir L, Fuchs CS, Kraft P, Stolzenberg-Solomon RZ, Jacobs KB, Arslan AA, Bueno-de-Mesquita HB, Gallinger S, Gross M: A genome-wide association study identifies pancreatic cancer susceptibility loci on chromosomes 13q22.1, 1q32. 1 and 5p15. 33. Nat Genet 2010, 42:224-228.

doi:10.1186/1471-2350-15-43

Cite this article as: Tang et al.: Identification of transcription factors and single nucleotide polymorphisms of $L r h 1$ and its homologous genes in Lrh1-knockout pancreas of mice. BMC Medical Genetics 2014 15:43.

\section{Submit your next manuscript to BioMed Central and take full advantage of:}

- Convenient online submission

- Thorough peer review

- No space constraints or color figure charges

- Immediate publication on acceptance

- Inclusion in PubMed, CAS, Scopus and Google Scholar

- Research which is freely available for redistribution 